\title{
A method for the delivery of reinforcement during exercise
}

\author{
KAREN J. COLEMAN, ROCCO A. PALUCH, and LEONARD H. EPSTEIN \\ State University of New York, Buffalo, New York
}

\begin{abstract}
The purpose of this study was to develop an automated system for positively or negatively reinforcing subjects while they exercised on an electronically braked bicycle ergometer. The circuitry used to interface the subject's pedal speed responses with the delivery of auditory reinforcement is presented, as well as a study designed to test the utility of the system. Twenty 18- to 25-year-old male subjects exercised on an electronically braked ergometer at a constant workload of $50 \mathrm{~W}$ for three 5-min blocks. During the first $5 \mathrm{~min}$, subjects pedaled at their preferred rate, and during the next two 5 -min blocks, they pedaled faster or slower than their baseline pedal speed to turn on a comedy tape for positive reinforcement, or turn off $90 \mathrm{~dB}$ of white noise for negative reinforcement. Circuits that interfaced the subject's pedal response with the delivery of reinforcement are discussed in detail. Both positive and negative reinforcement increased the targeted pedaling rates, with no differences as a function of targeting increased or decreased pedal speed. This system could be used with various reinforcers, such as video and other audio equipment to increase exercise behavior.
\end{abstract}

Physical activity has been shown to decrease many risk factors associated with chronic disease (Blair, Goodyear, Gibbons, \& Cooper, 1984; Kramsch, Aspen, Abramowitz, Kreimendahl, \& Hood, 1981; Paffenbarger \& Hyde, 1988; Paffenbarger, Laughlin, Gima, \& Black, 1970) and to provide many mental health benefits (Morgan \& O'Conner, 1988; Moses, Steptoe, Mathews, \& Edwards, 1989; Simons, McGowan, Epstein, Kupfer, \& Robertson, 1985; Steptoe \& Cox, 1988). In spite of this evidence, the majority of people in the United States do not engage in regular vigorous physical activity, and many are not active at all (Casperson, Christenson, \& Pollard, 1986; King \& Martin, 1993). This poor adherence may be due to the fact that sedentary people do not enjoy aerobic exercise (Wankel, 1993), and that the reinforcing properties of exercise are low, particularly in contrast to the high-reinforcing value of sedentary alternatives (Epstein, Smith, Vara, \& Rodefer, 1991; Vara \& Epstein, 1993). Many people exercise to avoid negative health outcomes, such as coronary heart disease or colon cancer. However, increasing exercise to avoid the negative consequences of a sedentary lifestyle may not lead to enjoyment of the exercise behavior, which in turn may result in poor exercise adherence. Increasing exercise behavior through the use of positive reinforcement, by providing positive reinforcers contingent upon increases in targeted exercise behaviors, may increase adherence of sedentary individuals to exercise programs and lead to long-term health benefits.

This study was funded in part by NIH Grant RO1 HD 25997. Correspondence should be addressed to L. H. Epstein, Behavioral Medicine Laboratory, Department of Psychology, State University of New York, Buffalo, NY 14260 (e-mail: lhenet@ubvms.cc.buffalo. edu).
Laboratory paradigms for altering exercise behaviors by the use of positive and negative reinforcement paradigms have been developed for animal research. For example, Buggy and colleagues (Burgess, Davis, Borg, \& Buggy, 1991; Garner, Terracio, Borg, \& Buggy, 1995) provided electrical brain stimulation or the avoidance of shock as positive and negative reinforcement procedures to increase exercising in rats. They demonstrated that running behavior was increased using both positive and negative reinforcement, but when animals were subjected to an endurance run without reinforcement contingencies, those rats that had been trained using positive reinforcement ran longer and more consistently than rats that had been trained to run with negative reinforcement (Burgess et al., 1991). Likewise, Drewett and Zbrozyna (1985) reinforced rats for exercising by providing contingent food or avoidance of shock. Animals who were positively reinforced for exercising showed lower elevations in blood pressure and heart rate in anticipation of exercise than did animals motivated to exercise with negative reinforcement. This research suggests that positively reinforcing exercise behavior in animals leads to better exercise performance and less physiological reactivity associated with exercise than negatively reinforced exercise behavior.

De Luca and Holborn (1992) described a method for delivering reinforcement to humans on the basis of subjects' pedal speed on a bicycle ergometer; however, their description of the apparatus used to deliver reinforcement did not provide enough detail to facilitate replication of their study. The purpose of this paper is to detail a system to deliver reinforcement to subjects during cycling exercise, dependent upon their pedal speed. All subjects cycled at the same relative workload to ensure that factors other than the experimental contingencies did not control behavior. 


\section{METHOD}

\section{Stimuli, Equipment, and Design}

All subjects exercised on an electronically braked Cateye EC1600 ergometer (Vacumetrics, Ventura, CA) that allowed workload to be held constant at any pedal speed. Input from the ergometer was relayed to the programming equipment by a magnet and magnet sensor connected to the ergometer. Reinforcement contingencies based on pedal speed were controlled in an adjacent lab room by Coulbourn modular components (Coulbourn Instruments, Lehigh Valley, PA). The specific components included one retriggerable one-shot (S52-12), three "and" gates (S31-12), three "or" gates (S32-12), one flip-flop (S41-12) with two separate inputs and outputs, two predetermining counters (S43-30), three universal timers (S53-21), one dual electronic counter/display (R11-45), one quad-buffered switch (S96-24), one white noise generator (S81-02), one programmable attenuator (S85-08), and one audio mixer power amplifier (S82-24). Subjects pedaled to listen to an amplified Bill Cosby stand-up comedy tape (positive reinforcement), or to avoid listening to $90 \mathrm{~dB}$ of white noise (negative reinforcement). Bill Cosby and white noise were delivered to each subject through headphones connected to an audiotape player.

Twenty male students, $18-25$ years of age, were recruited through SUNY Buffalo psychology department's mass testing pool to test the reinforcement delivery system. Subjects were administered the Physical Activity Readiness Questionnaire (Gordon \& Mitchell, 1993) to ensure they had no medical complications that would limit their ability to exercise, and received one credit for Psychology 101 for participating. Workload on the cycle ergometer was held constant at $50 \mathrm{~W}$, approximately equal to 3 METS. This is a light workload (Ainsworth et al., 1993), used to prevent the effects of workload on the motivational properties of the auditory reinforcers. For the first $5 \mathrm{~min}$ of each experimental session, all subjects were allowed to pedal at a speed with which they felt comfortable, and then they were randomly assigned to one of four groups that varied whether or not positive or negative reinforcement was presented first, and whether the targeted behavior was an increase or a decrease in pedal speed from the preferred baseline pedal speed. This design was used to compare the effects of positive and negative reinforcement on exercise behavior and to ensure that the contingencies would modify targeted pedaling rates above or below their usual pedaling rates.

\section{Programming}

Programming principles. Two threshold circuits were used to determine if the subject's pedal speed was above or below a specific range of pedal speeds. This range was referred to as the pedalspeed response bandwidth. The two threshold circuits were set to create lower and upper pedal speed limits of the response bandwidth: a lower threshold circuit for the lower boundary of the bandwidth and an upper threshold circuit for the upper boundary of the bandwidth. When a subject maintained a pedal speed faster than the lower boundary and slower than the upper boundary, he met the pedal speed contingencies and received auditory reinforcement through the headphones. The subject was required to maintain a pedal speed within the bandwidth for several pedal speed revolutions before the delivery of reinforcement. This ensured that the subjects had sustained a consistent pedal speed before receiving reinforcement and that the delivery of reinforcement was smooth and uninterrupted. This was accomplished by counting pedal revolutions within the bandwidth and delivering reinforcement when a predetermined number of revolutions in that range had been made by the subject.

Overview of programming. Figure 1 presents a detailed diagram of all circuits used in the delivery of auditory reinforcement during cycling, beginning with inputs from the magnet sensor on the ergometer and ending with auditory output to the headphones worn by the subject. The magnet sensor was connected to one of the ergometer's pedals, and a magnet was mounted at the base of the ergometer opposite the sensor. The sensor was connected to the programming equipment in another room with two leads. One lead was grounded and the other was connected to a one-shot so that each pedal revolution resulted in a pulse transmission. This one-shot operated reset timers that were used to detect pedal speeds above or below a lower threshold circuit and below or above an upper threshold circuit. Audio reinforcement was delivered through headphones to each subject contingent on his maintenance of a pedal speed within a pedal speed bandwidth defined by these lower and upper threshold circuits. Using a flip-flop and "and" gates, logic conditions were used to control an auditory attenuator set at $128 \mathrm{~dB}$. If the subject was to receive positive reinforcement when he maintained a pedal speed within the bandwidth contingency, he would turn the attenuator off and hear the Cosby tape through the headphones. If he received negative reinforcement by maintaining a pedal speed within the bandwidth contingency, he would turn the attenuator on and shut off the $90 \mathrm{~dB}$ of white noise coming through the headphones. The attenuation setting should be set for the loudest sound presented to the subject $(90 \mathrm{~dB})$; however, the attenuator was set to $128 \mathrm{~dB}$ in the present experiment to ensure that no sound came through the headphones when the attenuator was on.

Lower threshold for reinforcement. The lower threshold circuit for the delivery of reinforcement involved setting a pedal speed that each subject had to maintain or surpass in order to receive reinforcement. For example, if a pedal speed threshold of 60 revolutions per minute (rpm) was used, reinforcement could be activated if the subject pedaled $\geq 60 \mathrm{rpm}$, and it would be deactivated if he fell below $60 \mathrm{rpm}$. A lower threshold of $60 \mathrm{rpm}$ was accomplished by setting a timer to $1 \mathrm{sec}$ that was initiated at each pedal revolution. Any pedal speed could be set for the lower limit threshold circuit by adjusting this timer to more than $1 \mathrm{sec}$ (slower than $60 \mathrm{rpm}$ ) or less than $1 \mathrm{sec}$ (faster than $60 \mathrm{rpm}$ ). If the subject pedaled above $60 \mathrm{rpm}$, pedal revolutions occurred prior to the timer timing out, resetting the timer (still in the operate mode) and initiating the operation again. The initial operation of the timer also triggered a predetermining counter set at 5 . As long as the operation on the timer was initiated, the predetermining counter would continue to count down. If the subject pedaled slower than $60 \mathrm{rpm}$, the timer would time out, and an end pulse would reset the predetermining counter before it counted to 5 . If the counter reached 5 counts, auditory reinforcement was delivered to the subject. This was accomplished by sending the timer and counter operate pulses concurrently into an "and" gate that controlled a flip-flop. The flipflop was set to operate if the timer remained on, and the predetermining counter reached 5 . The flip-flop operate signal was then routed to the $128-\mathrm{dB}$ attenuator, with the attenuator output sent to an amplifier. The predetermining counter was used to ensure that subjects maintained a stable level of responding for $3 \mathrm{sec}$ before accessing reinforcement. Without this counter, reinforcement would have been delivered on a revolution-by-revolution basis, resulting in a "choppy" auditory output. Investigators may have to experiment with the best counter setting for the type of reinforcement delivered and the goals of their studies.

The output to the 128-dB attenuator determined whether the subject heard the comedy tape or did not listen to the white noise when his pedal speed was $\geq 60 \mathrm{rpm}$. The two reinforcement outcomes were accomplished using different outputs from the flip-flop. A " 0 " output from the flip-flop would turn the attenuator off and allow the comedy tape to come through the headphones (positive reinforcement). A "1" output from the flip-flop would turn on the attenuator and shut off the $90 \mathrm{~dB}$ of white noise coming through the headphones (negative reinforcement). The tape player remained playing throughout each reinforcement session, but the output from the tape player was run through the attenuator, ultimately determining what each subject heard on the basis of his pedal speed. 


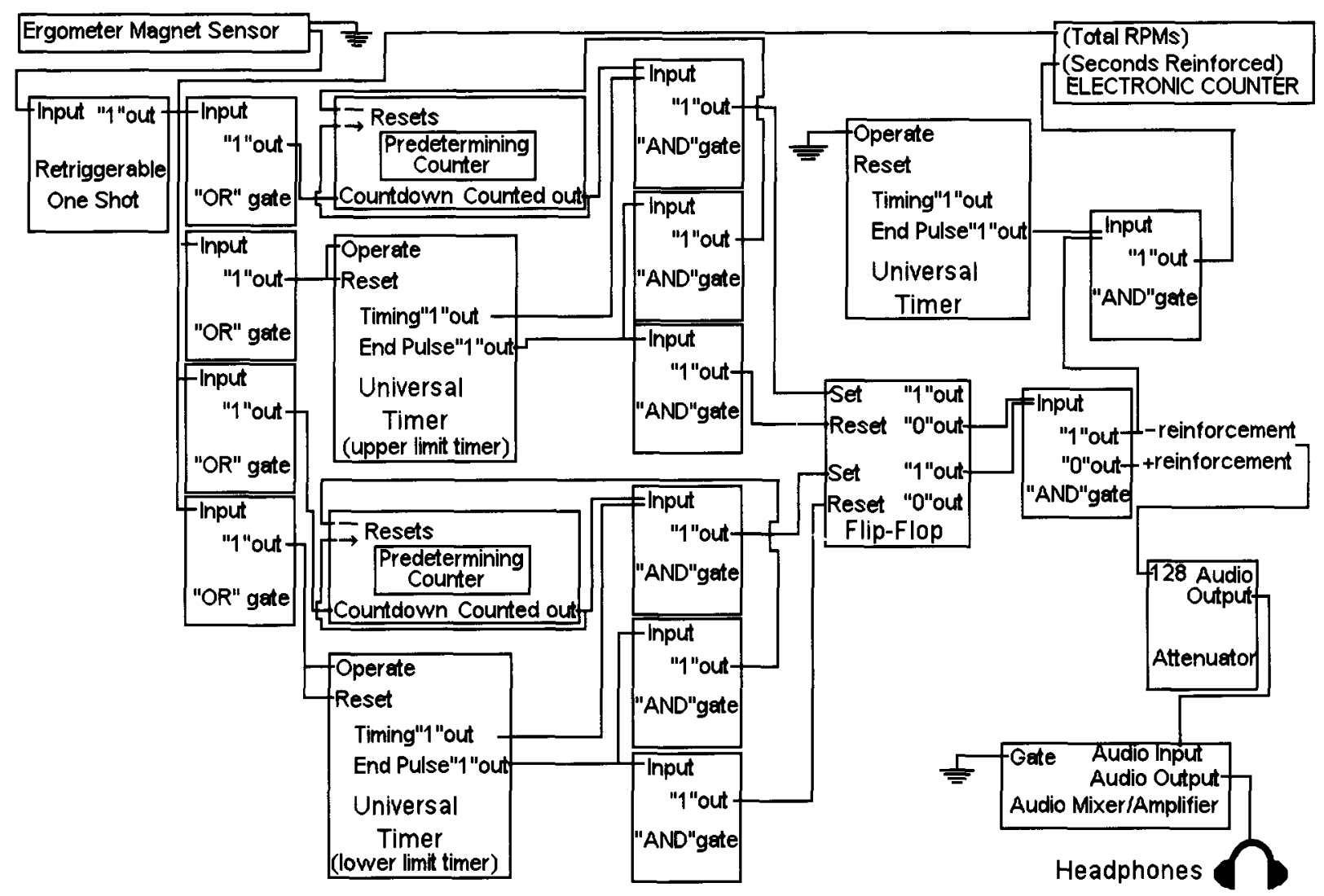

Figure 1. Detailed diagram of programming components involved in the delivery of reinforcement during exercise on a bicycle ergometer.

Adding an upper threshold for a reinforcement bandwidth. A narrow, 5-rpm bandwidth of pedal speed responding was chosen in the present study to create a more difficult reinforcement contingency than a single threshold (as detailed previously). This entailed adding an upper threshold circuit to the system described previously for a lower threshold circuit. With these two threshold circuits, subjects had to pedal equal to, or faster than, the lower threshold pedal speed, and slower than the upper threshold speed, to attain auditory reinforcement. For example, the subject may have had to pedal $\geq 60 \mathrm{rpm}$ (lower threshold) and $<65 \mathrm{rpm}$ (upper threshold) to access audio reinforcement. In addition to setting the lower limit timer to $1 \mathrm{sec}$, an upper limit timer would have been set to less than $1 \mathrm{sec}$ (approximately $0.92 \mathrm{sec}$ ) that was initiated at each pedal revolution if the subject pedaled $\geq 65 \mathrm{rpm}$. Any pedal speed could be set for the upper limit threshold circuit by adjusting this timer to more than $0.92 \mathrm{sec}$ (slower than $65 \mathrm{rpm}$ ) or less than $0.92 \mathrm{sec}$ (faster than $65 \mathrm{rpm}$ ). If the subject pedaled $\geq 65 \mathrm{rpm}$, pedal revolutions occurred prior to the timer timing out, resetting the timer (still in the operate mode) and initiating the operation again. If the subject pedaled slower than $60 \mathrm{rpm}$, the timer would time out, and an end pulse would reset a second predetermining counter. The first pedal revolution made by a subject initiated both predetermining counters.

Reinforcement delivery. If a pedal revolution occurred prior to the lower and the upper limit timers timing out ( $\geq 65 \mathrm{rpm}$ with the current contingency example), both timers would reset and remain in the operate mode. If a revolution occurred after both timers had timed out $(<60 \mathrm{rpm})$, an end pulse would reset both counters. Finally, if a revolution occurred before the lower limit timer timed out $(\geq 60 \mathrm{rpm})$ and after the upper limit timer timed out $(<65 \mathrm{rpm})$, the lower limit predetermining counter would continue to count to 5 and the upper limit predetermining counter would be reset by an end pulse. Operate pulses from the timer and counter of each threshold circuit were concurrently fed into separate "and" gates that each controlled a flip-flop. If the subject maintained a pedal speed $\geq 60 \mathrm{rpm}$ and $<65 \mathrm{rpm}$ for approximately $3 \mathrm{sec}$, the lower limit counter would count to 5 and the lower limit threshold flipflop would be set to operate, whereas the upper limit threshold flip-flop would be turned off, delivering audio reinforcement to the subjects. Outputs from both flip-flops were sent to one "and" gate. The type of reinforcement delivered depended upon the output from the "and" gate to the attenuator. Negative reinforcement resulted from an operate signal from the "and" gate, turning the attenuator on and shutting off the $90-\mathrm{dB}$ white noise coming through the headphones. Positive reinforcement resulted from turning off a signal from this "and" gate and allowing the comedy tape to be heard through the headphones.

The number of seconds spent receiving audio reinforcement was recorded for each subject using an electronic counter. A 1-sec pulse was sent from a grounded timer with the output from the reinforcement "and" gate to another "and" gate. Output from this "and" gate was routed to the electronic counter that displayed the total number of seconds each subject pedaled within the contingent bandwidth. Each pulse generated by the timer, while the subject received reinforcement, resulted in $1 \mathrm{sec}$ recorded by the counter. In addition, total number of revolutions made by each subject was recorded by a second electronic counter using output from the one-shot. 


\section{Reinforcement Procedures}

Each session began with a 5-min baseline period during which subjects were told to ride the bike for $5 \mathrm{~min}$ and pedal at a speed with which they were comfortable. During this baseline period, rpm were recorded in 30-sec intervals. Average baseline rpm were calculated and used to determine the reinforcement contingencies for each subject. If the target behavior to receive reinforcement was an increase in pedal speed, subjects had to pedal within a 5rpm bandwidth $10 \%$ faster than their baseline pedal speed. If the target behavior was a decrease in pedal speed, subjects had to pedal within a 5 -rpm bandwidth $10 \%$ slower than their baseline pedal speed.

After the 5-min baseline period, subjects were instructed that they would be riding the ergometer for 10 more minutes, divided into two 5-min blocks. Subjects were told which reinforcer they would receive at the beginning of the reinforcement exercise session, and after $5 \mathrm{~min}$ they were told they would receive the other reinforcer. They were not told the pedal speed they had to maintain to receive positive or negative reinforcement. Average rpm were recorded in 30-sec blocks, and time spent meeting the reinforcement criteria was also recorded for each condition. Each subject could have spent a maximum of $300 \mathrm{sec}$ listening to the comedy tape and $300 \mathrm{sec}$ escaping white noise.

\section{RESULTS}

The mean rpm difference from baseline pedal speed was calculated by subtracting each subject's rpm in the increase or decrease condition from their mean baseline $\mathrm{rpm}$. In addition, the percentage of the 5-min block spent within the positive and negative reinforcement contingencies for each subject was calculated. One mixed analysis of variance (ANOVA), with order of reinforcement (positive or negative first) and order of pedal speed (increase or decrease first) as between-subjects factors, and the type of contingency (increase/decrease) as the withinsubjects factor, was performed to assess differences in changes in pedal speed relative to baseline. Another mixed ANOVA, with order of reinforcement (positive or negative first) and order of pedal speed (increase or decrease first) as between-subjects factors, and the type of reinforcement (positive/negative) as the within-subjects factor, was performed to assess differences in the percentage of time receiving reinforcement.

There was a main effect of changing pedal speed relative to baseline $[F(1,16)=290.06, p<.001]$, with no main effects of either order of reinforcement or pedal speed. This is illustrated in Figure 2; pedaling rates are presented for each minute of the experiment within each condition: baseline, target of increasing pedal speed relative to baseline, and target of decreasing pedal speed relative to baseline.

There were no main effects or interactions in the analysis of time spent receiving reinforcement, indicating that regardless of the order of the reinforcement, order of the increase or decrease in pedal speed to receive reinforcement, or the type of reinforcement, subjects spent the same amount of time accessing positive $(72.4 \%)$ and negative $(71.2 \%)$ reinforcement. These results suggest that subjects met the targeted increasing or decreasing pedal speed contingencies, with both contingencies working equally well in controlling behavior.

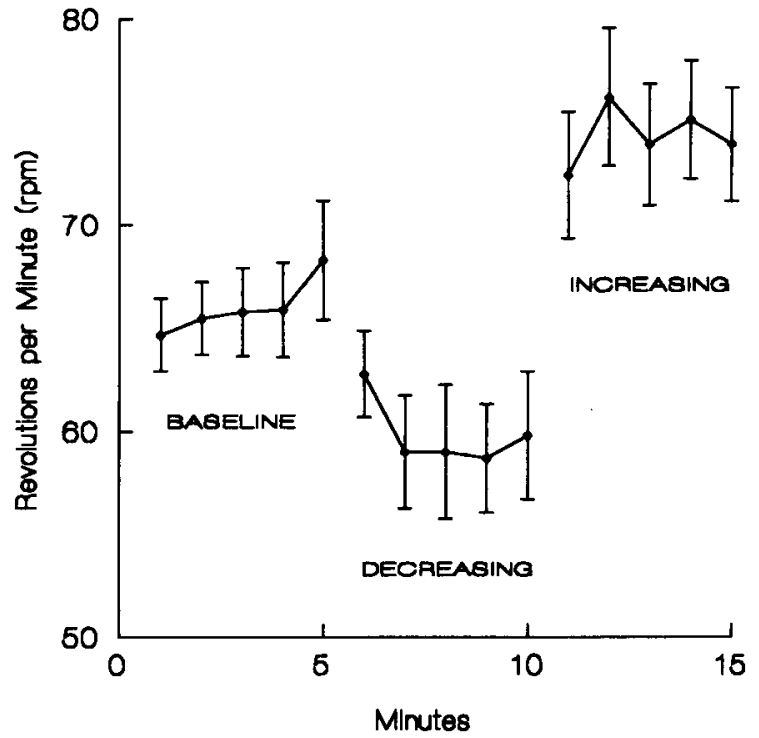

Figure 2. The mean revolutions per minute $(\mathrm{rpm}) \pm$ standard error are presented for each minute of each condition in the experiment. The first $5 \mathrm{~min}$ represent baseline $\mathrm{rpm}$; the second 5 min represent targeted decreasing pedal speed relative to baseline; and the last $5 \mathrm{~min}$, targeted increasing pedal speed relative to baseline. Means represented are independent of order of pedal speed or reinforcement.

\section{CONCLUSION}

This reinforcement procedure is a very flexible system that can be used to reinforce subjects for using exercise equipment and to determine the psychological and physiological effects of reinforcing exercise behavior in humans. We have described the methods for interfacing a tape recorder with an exercise bicycle to deliver audio reinforcement during exercise; however, a wide variety of exercise equipment or reinforcers could be adapted for exercise reinforcement using similar programming ideas. Among the uses for this system might be reinforcing exercise in naturalistic settings contingent upon access to various types of positive reinforcers. Eventually, the use of this system for reinforcing exercise behavior in humans may lead to increased adherence to an active and healthy lifestyle.

\section{REFERENCES}

Ainsworth, B. E., Haskel., W. L., Leon, A. S., Jacobs, D. R., JR. Montoye, H. J., Sallis, J. F., \& Paffenbarger, R. S., Jr. (1993). Compendium of physical activities: Classification of energy costs of human physical activities. Medicine \& Science in Sports \& Exercise, 25, 71-80.

Blair, S. N., Goodyear, N. N., Gibbons, L. W., \& Cooper, K. H. (1984). Physical fitness and the incidence of hypertension in healthy normotensive men and women. Journal of the American Medical Association, 252, 487-490.

Burgess, M. L., Davis, M. D., BorG, T. K., \& BugGy, J. (1991). Intracranial self-stimulation motivates treadmill running in rats. Journal of Applied Physiology, 71, 1593-1597.

Casperson, C. J., Christenson, G. M., \& Pollard, R. A. (1986). Sta- 
tus of the 1990 physical fitness and exercise objectives-Evidence from NHIS 1985. Public Health Reports, 101, 587-592.

De LuCA, R. V., \& HolborN, S. W. (1992). Effects of a variable-ratio reinforcement schedule with changing criteria on exercise in obese and nonobese boys. Journal of Applied Behavior Analysis, 25, 671679.

Drewett, P. N., \& Zbrozyna, A. W. (1985). Cardiovascular changes prior to exercise in appetitive and avoidance conditioning in rats. Physiology \& Behavior, 35, 529-532.

Epstein, L. H., Smith, J. A., VARa, L. S., \& Rodefer, J. S. (1991). Behavioral economic analysis of activity choice in obese children Health Psychology, 10, 311-316.

Garner, R. P., Terracio, L., Borg, T. K., \& Buggy, J. (1995). Intracranial self-stimulation motivates weight-lifting exercise in rats. Journal of Applied Physiology, 71, 1627-1631.

Gordon, N. F., \& MrTCHELL, B. S. (1993). Health appraisal in the nonmedical setting. In J. L. Durstine, A. C. King, P. L. Painter, J. L. Roitman, \& L. D. Zwiren (Eds.), ACSM's resource manual for guidelines for exercise testing and prescription (2nd ed., pp. 219-228). Philadelphia: Lea \& Febiger.

King, A. C., \& MarTIN, J. E. (1993). Exercise adherence and maintenance. In J. L. Durstine, A. C. King, P. L. Painter, J. L. Roitman, \& L. D. Zwiren (Eds.), ACSM's resource manual for guidelines for exercise testing and prescription (2nd ed., pp. 443-454). Philadelphia: Lea \& Febiger.

Kramsch, D. M., Aspen, A. J., Abramowitz, B. M., Kreimendahl, T., \& HooD, W. B., JR. (1981). Reduction of coronary atherosclerosis by moderate conditioning exercise in monkeys on an atherogenic diet. New England Journal of Medicine, 305, 1483-1489.
Morgan, W. P., \& O'Conner, P. J. (1988). Exercise and mental health. In R. K. Dishman (Ed.), Exercise adherence: Its impact on public health (pp. 91-121). Champaign, IL: Human Kinetics.

Moses, J., Steptoe, A., Mathews, A., \& Edwards, S. (1989). The effects of exercise training on mental well-being in the normal population: A controlled trial. Journal of Psychosomatic Research, 33, 47-61.

Paffenbarger, R. S., JR., \& Hyde, R. T. (1988). Exercise adherence, coronary heart disease, and longevity. In R. K. Dishman (Ed.), Exercise adherence: Its impact on public health (pp. 41-74). Champaign, IL: Human Kinetics.

Paffenbarger, R. S., Jr., Laughlin, M. E., Gima, A. S., \& Black, B. A. (1970). Work activity of longshoremen as related to death from coronary heart disease and stroke. New England Journal of Medicine, 282, 1109-1114.

Simons, A. D., McGowan, C. R., Epstein, L. H., Kupfer, D. J., \& RoBERTSON, R. J. (1985). Exercise as a treatment for depression: An update. Clinical Psychology Review, 5, 553-568.

STEPTOE, A., \& Cox, S. (1988). Acute effects of aerobic exercise on mood. Health Psychology, 7, 329-340.

VARA, L. S., \& EPSTEIN, L. H. (1993). Laboratory assessment of choice between exercise and sedentary behaviors. Research Quarterly for Exercise \& Sport, 64, 356-360.

WANKEL, L. M. (1993). The importance of enjoyment to adherence and psychological benefits from physical activity. International Journal of Sport Psychology, 24, 151-169.

(Manuscript received July 28, 1995;

revision accepted for publication November 7,1995 .) 